\title{
Le bio s'use-t-il ? Analyse du débat autour de la conventionalisation du label bio
}

Is organic quality wearing thin? Analysis of the debate around the organic label "conventionalisation"

\section{Geneviève Teil}

\section{(2) OpenEdition}

\section{Journals}

Édition électronique

URL : http://journals.openedition.org/economierurale/3708

DOI : 10.4000/economierurale.3708

ISSN : 2105-2581

\section{Éditeur}

Société Française d'Économie Rurale (SFER)

Édition imprimée

Date de publication : 30 novembre 2012

Pagination : 102-118

ISSN : 0013-0559

Référence électronique

Geneviève Teil, « Le bio s'use-t-il ? Analyse du débat autour de la conventionalisation du label bio », Économie rurale [En ligne], 332 | novembre-décembre 2012, mis en ligne le 30 novembre 2014, consulté le 01 mai 2019. URL : http://journals.openedition.org/economierurale/3708 ; DOI : 10.4000/ economierurale.3708 


\section{Le bio s'use-t-il ?}

\section{Analyse du débat autour de la conventionalisation du label bio}

Geneviève TEIL • Institut national de la recherche agronomique (INRA), Sciences pour l'action et le développement, France, et Institut national de la recherche agronomique (INRA) Centre Régional de Recherche Agronomique de Tanger, Maroc

a notion de durabilité tire sans doute son succès de son ambiguiité. Comment savoir en effet ce qui est durable et ce qui ne l'est pas ? Y a-t-il un seuil au delà duquel un état ou une démarche peut être considérée comme durable ? À partir de quelle durée une démarche est-elle durable ? De plus l'examen de la rémanence d'un objet oblige à en préciser la nature : qu'est-ce que le bio, quelles évolutions et adaptations peuvent être considérées comme tolérables ou inacceptables ? Comment peut-on répondre à de telles questions ? La notion même de durabilité a-t-elle un sens ?

La logique inciterait à tourner rapidement la page ; la prudence souligne qu'elle alimente de très nombreuses conversations, activités, occupe de nombreuses pages et personnes. Une notion vide de sens n'auraitelle pas déjà lassé ?

Cet article propose de regarder comment les acteurs font pour s'accommoder d'un objet si délicat. Il écarte la notion de durabilité en général (sujet trop vaste pour un si petit article) pour se centrer plutôt sur la crainte - récurrente chez certains agrobiologistes - de l'usure du label bio, de la perte de sa capacité à faire une différence entre des produits agricoles « conventionnels » et des produits agricoles « biologiques » et donc de son impossible durabilité. Comment et pourquoi le bio ne serait-il pas durable ? Quels sont les signes, les épreuves, les faits qui permettent de le craindre ?

Nous commençons tout d'abord par retracer la naissance de cette crainte née dans les milieux agrobiologistes au moment de l'instauration du label AB qui regroupe une variété de démarches afin de mieux diffuser l'agrobiologie. Cette crainte est entendue par les chercheurs qui se sont interrogés sur sa réalité sans conclure. Puis, nous nous tournons vers les acteurs et leurs débats à ce sujet afin de voir comment ils parviennent à concilier les deux éléments assez contradictoires que sont l'adaptabilité et la pérennité du bio. L'examen des débats permet de montrer le rôle inattendu que joue cette crainte dans la durabilité du bio.

\section{La crainte de l'érosion du label $A B$}

En 1981, les agrobiologistes qui cherchent à promouvoir toujours mieux le bio demandent et obtiennent des pouvoirs publics la reconnaissance d'une agriculture «n'utilisant pas de produits chimiques de synthèse ». De plus, les associations et marques commerciales privées qui promeuvent le développement d'agricultures bio se voient offrir la possibilité de faire homologuer leurs cahiers des charges et leurs « mentions » ou interprétations du bio, leur conférant, de ce fait, une sorte de validation officielle à leur démarche.

Le premier cahier des charges homologué, celui de Nature \& Progrès ne s'impose pas comme «Le » cahier des charges de la bio. Parallèlement à l'homologation des 14 cahiers des charges et mentions alors existants et pour éviter une dispersion du mouvement bio en une multitude de cahiers des charges différents, se négocie un label français unique.

Sa mise en place amène l'ensemble des bio à rechercher une définition consensuelle, reposant essentiellement sur le rejet de l'in- 
novation technique de l'agronomie « chimique ». Car depuis Fritz Haber l'industrie chimique a développé quantité de solutions aux problèmes de croissance des plantes, maladies, ravageurs, concurrence avec les mauvaises herbes. L'efficacité des pesticides, fongicides et herbicides augmente les rendements, accompagne le changement des modes de vie des agriculteurs qui veulent eux aussi pouvoir prendre des vacances par exemple. Mais ce succès technique transforme aussi l'agriculture qui repose sur une reconstruction toujours mieux maîtrisable et donc de plus en plus « artificialisée » des processus biologiques, isolée et réduite à un processus de production de denrées alimentaires aux caractéristiques fortement prédéfinies.

Le règlement du label $\mathrm{AB}^{1}$ grâce auquel les bio cherchent à promouvoir une agriculture alternative consiste essentiellement dans la prohibition des engrais et produits de traitement de synthèse ; il accapare la dénomination «bio » et connaît un grand succès. Des 14 cahiers des charges et chartes privées de production biologique qui lui préexistaient n'en restent que deux, celui de Nature \& Progrès $^{2}$ pour le bio, qui entame une «traversée du désert » suite au départ de nombre de ses adhérents producteurs comme consommateurs, et Déméter pour la biodynamie.

\footnotetext{
1. Le label $\mathrm{AB}$ est associé à un cahier des charges français, le CCREPABF plus exigeant que le cahier des charges du label bio européen en particulier pour ce qui concerne l'élevage.

2. Pour être tout à fait précis, il faudrait dire 3 , car le cahier des charges de SIMPLES centré sur la cueillette de simples a lui aussi persisté. Nature \& Progrès et Déméter sont en France les deux principales associations de défense du bio ; elles proposent des cahiers des charges privés en marge du label public $\mathrm{AB}$ français devenu européen à la suite de la suppression de la subsidiarité européenne en matière de certification bio. Les deux associations regroupent des producteurs, distributeurs et consommateurs tandis que la FNAB, Fédération nationale de l'Agriculture Biologique est un syndicat de producteurs certifiés $\mathrm{AB}$ uniquement.
}

Cependant, une crainte se développe dans l'ombre de ce succès. Des producteurs ou responsables d'associations pointent la menace qui surgit de l'absence de la dimension socio-économique alternative dans la réglementation du label et le recours, volontaire ou non, de certains producteurs aux circuits habituels de commercialisation :

"Ce qu'on peut dire aujourd'hui, c'est que l'institutionnalisation de la bio a marqué ses limites (ou ses objectifs) ; la bio est actuellement dans une phase de "récupération marchande" : certains voudraient limiter son développement à un copiercoller de la production et de la distribution des produits alimentaires conventionnels. »

(Harrouch, 2003)

Ils pointent du doigt en particulier la grande distribution, la pression sur la productivité et les prix qu'elle fait subir aux producteurs et qui ne peut aboutir selon eux qu'à une érosion des exigences du bio. C'est cette même pression économique qui est selon eux la cause majeure de la transformation récente et des excès de l'agronomie conventionnelle contre lesquels ils se sont dressés et que des interprétations tronquées du bio, restreintes aux exigences réglementaires purement techniques du label, sont en train de ramener au cœur même du mouvement.

\section{La contribution de la recherche à l'érosion du label}

Des chercheurs se sont eux aussi inquiétés d'une telle évolution. Deux articles de recherche parus dans la même livraison de la revue Sociologia Ruralis, au raisonnement bien différent mais souvent cités ensemble (Buck et al., 1997) (Tovey, 1997) concluent tous deux à la mission impossible du bio.

Buck et ses co-auteurs (op. cit.) notent en Californie un rapprochement entre agriculture conventionnelle et bio (organic en anglais). Ils invoquent pour l'expliquer, la mécanique même de l'économie de marché et l'insuffisance des barrières posées par 
les bio à l'entrée de ce segment de marché très profitable. Les producteurs « conventionnels » attirés par ce mode de production bio, se convertissent et importent une rationalisation de la production à grands coups de capitaux investis. Les marges confortables dont ils profitent leur permettent de faire pression sur les prix et induisent aussi une augmentation de la valeur du foncier qui chasse petit à petit les pionniers du bio en général peu dotés en capital. De plus, la demande est plus intéressée par des qualités propres des produits bio (santé, environnement, goût...) que des questions d'organisation et d'accès aux marchés ; elle n'oppose donc guère sinon aucune résistance à la restructuration de la production biologique et l'abandon des principes socio-économiques alternatifs durables qui fondaient le mouvement bio d'origine.

Dans le second article, Tovey pointe un mécanisme institutionnel : les pouvoirs publics irlandais utilisent les subsides européens des mesures agri-environnementales pour favoriser le développement du bio sur la seule base des principes agronomiques de la certification bio à l'exclusion de tout objectif socio-économique. Ils induisent ainsi une croissance du bio qui déborde la vision socio-économique contestataire initiale et lui substitue une simple variante de l'agronomie classique qui utilise des produits naturels au lieu de produits de traitement et fertilisation chimiques.

Loin de faire l'unanimité, la publication de ces articles occasionne un vif débat repérable sous le nom de « conventionalisation », nom donné à la logique implacable d'érosion du bio et ses quelques mécanismes identifiés d'appropriation, de « commodification » ou de «bifurcation ». En effet, l'idée d'une logique capitaliste de capture de la valeur évoquée par Buck, Getz et Guthman est retournée au nom de la capacité du capitalisme à toujours intégrer ses propres contradictions pour poursuivre son développement et donc sortir de ses propres impasses (Coombes et Campbell, 1998).
Guthman persiste en invoquant les faiblesses de la signalisation du bio et son incapacité à imposer une qualité bio non seulement agronomique, mais aussi socio-économique $^{3}$. Début 2001, paraît un numéro spécial de Sociologia Ruralis (Michelsen, 2001) consacré à la conventionalisation. Il élargit le débat jusque-là restreint à l'économie pour opposer à la conventionalisation une série de logiques de forces sociales concurrentes. Et pour tenter de sortir de l'affrontement disciplinaire, il apporte aussi la contribution de travaux empiriques qui inaugurent une série d'études de cas (Coombes et Campbell, op. cit. ; Dantsis et al., 2009) et de «mesures » de la conventionalisation (Hall et Mogyorody, 2001 ; Lockie et Halpin, 2005).

Les études de cas montrent que l'usure n'est pas aussi forte partout. Dans certains pays comme la Nouvelle Zélande, le bio « véritable » semble bien résister à côté d'une version industrialisée et affadie. Les auteurs de ces études de cas contestent donc l'universalité des forces érosives inventoriées jusque là mais sans l'expliquer.

La mesure des forces conventionalisatrices quant à elle, ne va pas sans difficulté. Pour apprécier l'effet de transformation du bio, les auteurs comparent les « valeurs » des nouveaux agriculteurs biologiques, en particulier ceux qui se convertissent, à celles des pionniers. Mais ils sont contraints dans cette comparaison de considérer que les pionniers sont comme des dinosaures fossilisés, d'unanimes témoins d'autres temps particulièrement rigides. Comme le note Best (2008) à la suite de Darnhofer (2006) ou Tovey (op. cit.), il faudrait pouvoir disposer d'une définition plus précise de la conventionalisation, car il n'est pour l'heure pas possible de distinguer ce qui, dans les changements observés, relèverait d'une appropriation du bio par le conventionnel ou,

3 L'argument de Guthman est très proche de la crainte de "récupération marchande" évoquée plus haut. 
à l'opposé, du propre développement du bio et de son adaptation à des situations toujours nouvelles et changeantes.

Face à ces difficultés, Darnhofer et al. (2010) suggèrent de rapporter les pratiques à ce que les auteurs considèrent être « $\mathrm{La}$ » définition du bio. L'article s'appuie ainsi sur les quatre principes listés par l'International Federation of Organic Agriculture Movement (IFOAM, non daté) comme formant les racines de l'agriculture biologique - et de suivre l'ajustement des pratiques à ces principes grâce à des batteries d'indicateurs.

Or, ce parti pris du choix de la définition de l'IFOAM oublie la dimension historique de toute élaboration de principes. Elle fait aussi fi de la diversité des interprétations du bio, en particulier de tous ceux qui ne se reconnaissent pas dans l'IFOAM ou trouvent ces principes insuffisants. L'IFOAM qui entend accompagner le développement international du bio s'appuie sur des critères consensuels qui excluent notamment les dimensions socio-économiques alternatives. Ainsi la prohibition du recours à la grande distribution ne fait-elle pas partie des critères retenus dans l'article alors que c'est un point essentiel pour nombre d'entre eux ${ }^{4}$.

Une autre voie explorée par des sciences humaines plus compréhensives prend plus au sérieux la diversité des interprétations des acteurs. Les chercheurs qui la suivent, tentent d'analyser la conventionalisation non plus comme un jeu de forces cachées s'imposant aux acteurs, mais comme le fruit de divergences d'interprétation du bio et de sa durabilité. La théorie des conventions sur laquelle s'appuient plusieurs de ces travaux (Murdoch et Miele, 1999; Campbell et Liepins, 2001 ; Rosin et Campbell, 2009) transforme la conventionalisation, usure du

4. On peut aussi noter que tout comme le « biorecette ", l'article réduit l'agriculture biologique à des indicateurs de pratiques, ce que les bio alarmés pointent comme étant la cause même de la menace d'érosion du bio. bio par le système agricole rationalisé, en un jeu d'oppositions entre grammaires idéologiques ou conventions de valeurs. Cette interprétation du bio comme valeur le transforme à l'exact opposé des travaux précédents qui cherchaient à lister les critères permettant d'en saisir le contenu objectif, en une simple construction subjective. La menace d'usure du label devient dès lors une simple autosuggestion performative ${ }^{5}$.

Pourtant les acteurs ne vivent pas que dans un royaume d'idées qui dicteraient leur loi aux plantes, champs et chiffre d'affaires indépendamment de l'expérience quotidienne qu'ils peuvent en faire. Plutôt que de poursuivre dans l'une des deux voies objective ou subjective précédentes, nous avons préféré revenir aux acteurs et au problème qu'ils pointent. Contrairement aux travaux précédents, nous ne nous sommes pas limités aux valeurs ou idéologies en tant que principes moraux subjectifs apposés à l'expérience que les acteurs font du monde. Dans la lignée des études sociotechniques (Latour, 2007) ou pragmatiques (James, 1996) nous avons ainsi cherché à cerner la menace que pressentent certains, à travers l'expérience qu'en ont les acteurs, des signes qui la manifestent, des accusations qu'ils portent contre certains de leurs collègues, des réponses qui leur sont faites dans le but de comprendre ce qui fait la différence entre le bon et le mauvais bio qui le menace. Nous avons ainsi réalisé une analyse pragmatique qui fait du bio, comme de son objectivité, le résultat de l'expérience collective - mais non nécessairement partagée.

L'analyse présentée ici est en partie issue d'un projet mené pour le ministère de l'Ecologie par une équipe de chercheurs, Barrey, Blanchemanche, Charpigny, Floux, Hennion

5. La difficulté qui s'ensuit est connue. Il est impossible de prévoir lesquelles des ces forces objectives ou subjectives vont induire une conventionalisation d'un label donné, et par conséquent incapable de décider le cas échéant les bonnes mesures de prévention. 
et nous-mêmes au sujet de la qualité environnementale des vins (Teil et al., 2011). L'étude consacrée au bio s'est appuyée sur plus de 70 entretiens avec des vignerons en bio ou biodynamie certifiés (62) ou non certifiés (10) installés en Val de Loire, Jura et Languedoc-Roussillon, des employés des pouvoirs publics ou de centres techniques en charge du bio, des distributeurs bio, des consommateurs, des journalistes, des organismes de certification...

Tableau 1. Répartition des entretiens par domaine d'activité

\begin{tabular}{l|c}
\hline Domaine d'activité & $\mathbf{N b}$ \\
\hline Production & 115 \\
\hline Distribution/Nente & 36 \\
\hline Administration centrale & 12 \\
\hline Technique\&Recherche & 14 \\
\hline Syndicat agricole & 7 \\
\hline Certification de qualité & 15 \\
\hline Média & 10 \\
\hline Restauration & 3 \\
\hline Demande & 13 \\
\hline Industrie phytosanitaire & 6 \\
\hline Total & $\mathbf{2 3 1}$ \\
\hline
\end{tabular}

Tableau 2. Répartition des entretiens selon la région

\begin{tabular}{l|c}
\hline Région & $\mathrm{Nb}$ \\
\hline Languedoc-Roussillon & 80 \\
\hline Val-de-Loire & 98 \\
\hline Paris & 51 \\
\hline Autres (Jura) & 2 \\
\hline Total 231 & \\
\hline
\end{tabular}

La liste détaillée des entretiens est présentée en annexes. Nous avons complété cet ensemble par sept entretiens auprès de représentants et employés d'associations pour la promotion du bio et de la FNAB et quatre demi journées d'observation participante. À ce corpus d'entretiens s'ajoute également un important corpus de données textuelles -rassemblant divers articles, revues et ouvrages, pages de blogs ou de sites internet, rapports de recherche ou d'institutions publiques ou privées - lié aux questions environnementales en viticulture, mais aussi plus généralement en agriculture, car les qualifications ne s'y restreignent pas.

\section{L'érosion du label une menace contestée}

Le label $A B$ a suscité des débats puis des rejets dès son instauration en 1991. Des rejets individuels au départ, puis un boycott par l'association Nature \& Progrès en 1995. Peu suivi à l'époque, il connaît depuis 2000 un regain d'adhésion; le nombre d'adhérents «boycotteurs » qui se certifient exclusivement Nature \& Progrès et refusent la certification $\mathrm{AB}$ est passé d'un tiers à la moitié des adhérents de l'association en 2010. La vérité de la menace se diffuserait-elle doucement? Mais dans ce cas, pourquoi les autres résistent-ils ? Cette section présente l'expérience qui amène les dénonciateurs de l'usure à mettre en cause un certain usage du label $\mathrm{AB}$ et la réponse qui leur est faite.

\section{Dépossession et réappropriation du bio}

Les détracteurs mécontents du label ${ }^{6}$ parlent d'institutionnalisation de la bio : certains se disent « dépossédés » par le label AB de la vigilance qu'ils exerçaient sur l'agriculture biologique, ses mises en œuvre, ses diverses interprétations ou innovations ; un sentiment à nouveau fortement ressenti en $2010 \mathrm{avec}$ la publication du nouveau règlement du bio qui supprime la subsidiarité française.

"... le cahier des charges européen c'est un texte consensuel et qui pour certains a sonné un peu la perte du contrôle des principes fondamentaux de l'agriculture biologique. [...\&... Il] est discuté maintenant loin des acteurs de la bio, il va nous échapper. Il est un peu voué à un nivellement par le bas parce qu'issu d'un consensus large et avec l'élargissement de l'Europe c'est encore plus vrai. "

(Entretien association Nature \& Progrès : $1 \& 2^{7}$ )

6. Nous rapportons ici la critique envers le label $A B$ faite par des militants d'associations dont au premier rang Nature \& Progrès et des producteurs bio, souvent eux aussi militants d'associations. Pour ne pas trop alourdir le texte, nous ne l'avons pas rappelé à chaque phrase.

7. Les chiffres indiquent les numéros de page des entretiens tous retranscrits sous un format normalisé. 
Les membres des associations discutent les enjeux du bio et les bonnes et mauvaises pratiques ; ils en ajustent le concept et révisent périodiquement les cahiers des charges pour tenir compte d'expériences nouvelles, d'innovations intéressantes ou indésirables... Or, avec le label, le bio est confié d'une part, à un organisme de contrôle indépendant et d'autre part, à la sagacité des usagers consommateurs de bio, dont les producteurs inquiets doutent de la compétence ou de l'expertise. Ils trouvent que leur intérêt pour le bio se limite à la prohibition des produits chimiques « non naturels »; qu'ils ne s'intéressent guère aux cahiers des charges ou aux débats soulevés par les pratiques. Les acheteurs « font confiance», autrement dit, ils délèguent au label l'identification des produits bio. Les consommateurs s'excluant du débat, la discussion critique de ce qu'est le bio et son contrôle repose donc au jour le jour sur les choix et les jugements des employés ou membres des organismes certificateurs ${ }^{8}$.

Deux exemples reviennent pour souligner les manques de ce contrôle :

- l'agriculture productiviste qui n'utilise que des produits naturels de traitement; - les cultures sans produits de synthèse mais « hors sol ».

Tous deux sont jugés trahir l'idée du bio tout en respectant au mieux le cahier des charges du label. Ces deux exemples sont cités comme le parangon d'une récupération du bio par les gros opérateurs ou la « logique capitaliste » de marché. Comme le label n'introduit aucun critère économique, les procédures de contrôle sont jugées incapables d'empêcher la perversion du bio qu'elles induisent.

Ces interprétations du bio sont souvent qualifiées de «bio-recette » : comme une

8. Cette dénomination inclut l'ensemble de ce que l'on peut appeler la « sphère du contrôle » avec ses commissions de contrôle, comités consultatifs, ... et que les acteurs nomment souvent "le contrôle" sans plus de précision. recette, elles s'appuient sur l'explicitation d'ensemble de techniques bio, mais auxquelles il manque «l'esprit» du bio, une analyse plus large, plus contextualisée, qui en visant un but - le développement d'une agriculture alternative pérenne parce que protégée des forces marchandes néfastes - réintroduit les dimensions socioéconomiques.

Pour anticiper la dérive redoutée, les deux associations Déméter et Nature \& Progrès révisent leurs cahiers des charges, chartes et principes directeurs ${ }^{9}$. Les contraintes agronomiques sont renforcées et les principes d'une agriculture durable tant du point de vue de la nature que des humains, explicités. Difficiles sinon impossibles à traduire ou border par des critères socio-économiques testables, ils sont inscrits dans les chartes comme un engagement des agriculteurs dont le contrôle est assuré par des procédures internes de garantie participative : les membres des associations assurent eux-mêmes une vigilance de l'interprétation, de la mise en œuvre et de l'engagement du producteur ou du transformateur dans le bio ou la biodynamie par les autres membres de l'association.

"Le travail en biodynamie exige que l'on se sente
fortement relié à l'essence de la méthode biody-
namique, à ses principes et à ses buts. À cet effet,
il est nécessaire de pénétrer les processus naturels,
en utilisant l'observation, le processus de pensée
et la perception. Une connaissance toujours plus
approfondie des liens dans la nature, peut être
acquise par des efforts continus. Le travail en
commun dans les différentes associations, les mani-
festations publiques, les revues et les livres sont des
sources importantes d'aide et de soutien. Cepen-
dant, si quelqu'un ne veut utiliser ces normes que
comme on le fait souvent avec les lois, c'est-à-
dire, en se ne préoccupant de ne s'attacher qu'au
côté formel ou en cherchant des échappatoires

9. La suppression du cahier des charges français et son remplacement par le cahier européen a suscité une réaction similaire avec la création de la marque Bio-Cohérence. Son cahier des charges est en effet plus exigeant que celui du label $\mathrm{AB}$ et tente d'intégrer des critères socio-économiques pour empêcher le « détournement » du bio. 
parce qu'il y a économiquement avantage, il ferait mieux de pratiquer un autre type d'agriculture. C'est la tâche de l'association Déméter France, de ses représentants, des conseillers d'empêcher que les choses ne prennent cette tournure. »

(Déméter, 2004)

\section{Le label pour diffuser le bio}

Pour un grand nombre d'adeptes, le bio est un état d'esprit, une autre façon de penser la production agricole. Celle-ci ne cesse de se discuter dans les multiples instances collectives formant le « réseau » de la Fédération nationale de l'agriculture biologique (FNAB). Mais tous ne rejettent pas le label, loin s'en faut.

Pour certains, la protection de l'environnement est bien une question de moyens et de proscription des mauvaises pratiques. Dès lors que les produits de synthèse sont interdits, l'essentiel est fait; l'agriculture redevient une agriculture non polluante, saine, respectueuse de l'environnement et du consommateur. Le marché n'est donc pas à craindre, mais à utiliser pour une tâche essentielle : développer le bio, le généraliser. Et de ce point de vue, le label offre des avantages considérables, il permet de faire connaître les produits bio, de les amener partout où portent les marchés, d'augmenter sa crédibilité en en finissant avec les interprétations rivales.

Pour ces adeptes du label AB, les surenchères qualitatives et les chartes pour le développement de nouvelles relations socioéconomiques ne font que confiner le bio à des « niches » réservées à des élites seules capables d'acheter ces produits jugés très chers. Ils veulent au contraire contribuer à diffuser le bio et le rendre « ordinaire ». Ils se moquent de ses circuits de diffusion inefficaces qui font monter le prix des produits comme plus tard de ces «bobos » parisiens qui détournent le bio pour en faire un produit de luxe avec la complicité, bien sûr, des cahiers des charges privés très exigeants des associations. Ils rejettent fermement ces stratégies d'enfermement et utilisent au contraire toutes les ressources offertes par les marchés pour diffuser leurs vins partout. Il est ainsi essentiel pour eux de mettre sur le marché des vins à des prix comparables aux autres pour qu'ils ne souffrent d'aucune discrimination.

Un premier producteur de notre enquête a « rationalisé » et « optimisé » son appareil de production, ajouté des activités de négociant à celles de producteur et adapté l'étiquetage de son vin aux demandes de ses clients. Une de ses cuvées pour la NouvelleZélande s'appelle « bin » à la manière d'un célèbre négociant australien. Un second producteur a misé sur les économies d'échelles et monté une exploitation viticole de 220 hectares certifiée AB, pratiquant la biodynamie. Tous deux distribuent en grande surface, vendent et exportent leurs vins à des tarifs semblables à ceux des autres vins de l'appellation pour faciliter au mieux la diffusion de leurs produits. Ils expliquent comment, contrairement à l'immense majorité des bio, ils se plient aux exigences de volume, de standardisation et de régularité de la grande distribution ou des grands négociants à l'export où ils profitent de la reconnaissance et de la crédibilité du label $\mathrm{AB}$ à l'étranger, ce qui les décharge du poids de la commercialisation de leurs produits.

Ces producteurs étaient bien seuls dans les années 1980 (deux sur sept producteurs sous certification privée avant 1990 dans notre échantillon) à dénoncer la «peur irrationnelle des marchés » qu'ils voyaient chez leurs collègues, et leurs relations étaient parfois houleuses. Mais ils sont devenus moins marginaux avec l'arrivée de nouveaux certifiés très préoccupés d'environnement et convaincus, eux aussi, que le rejet des produits de synthèse amène une solution radicale au problème des pollutions agricoles. Ils sont donc de ce fait moins ou peu engagés dans la réflexion étendue de l'agronomie stricto sensu à l'économie de la production agricole et à sa commercialisation. Peu soucieux des travers de l'économie de marché, ils sont plus préoccupés de parvenir à assurer la bonne santé écono- 
mique de leurs entreprises, une arme essentielle, selon eux, du développement du bio.

Deux interprétations du bio, assez divergentes, se développent donc. Dans l'une, le bio est un objet en élaboration, tendu vers un but, celui d'une agriculture durable tant du point de vue de la nature que de ses habitants, et qui réfléchit en continu ce qu'il est, repense sans cesse ses résultats et ses moyens. Dans l'autre, le bio est un objet suffisamment défini par un ensemble de pratiques et d'interdits. La question du contrôle reste aussi cruciale pour les uns et les autres, car ils ne comptent pas sur les consommateurs pour assurer une quelconque vigilance : contrairement à la qualité gustative des vins, la qualité du bio n'est pas vérifiable directement par le consommateur. Le sérieux du contrôle est un des points incontournables de la commercialisation et de la diffusion du bio.

Bio militants des associations et bios partisans du marché n'ont pas la même vision de la mise en œuvre du bio. Pour les premiers, les critères du label ne forment qu'un cadre minimal d'interprétations dont « l'esprit» doit être discuté. Pour les seconds au contraire, ils définissent et garantissent le bio. Ces derniers ne se sentent pas « dépossédés » du bio par le label qui «n'appartient » à personne. Ce ne sont pas pour autant des sympathisants tièdes. Le bio est un mode de production auquel ils adhèrent et comme les autres, aspirent à développer ; ils sont d'ailleurs parfois très actifs dans le milieu associatif bio. L'un des deux producteurs de notre échantillon avait lancé un concours des vins bio pour tenter d'améliorer la qualité gustative des vins qu'il pensait être un handicap à son développement.

Les deux ne se différencient pas tant par leur acceptation ou leur rejet du marché que par la modalité d'élaboration du bio qu'ils soutiennent. Pour les partisans du marché, le bio est une qualité circonscrite, définie et normalisable d'un objet. Ce qui, dans la production de l'objet ne relève pas de la norme, fait partie des « autres » qualités du produit et relève au contraire des choix du producteur. Ils s'opposent ainsi à ceux qui voient dans le bio au contraire une qualité globale dont l'évaluation doit toujours être repensée.

\section{Une coexistence parfois houleuse}

Les relations entre les tenants des deux partis sont assez conflictuelles. Chacun se renvoie une interprétation du bio, «bioniche » contre « bio-business », qui met en cause la sincérité ou l'efficacité de l'engagement de l'autre. Pour éviter de reprendre ces appellations trop péjoratives, nous appellerons les premiers les bio éco-alternatifs pour insister sur le caractère étendu et global de leur interprétation et les seconds les « bio label » qui délèguent au label le portage de l'identité bio.

Les éco-alternatifs de notre échantillon craignent que le marché à qui l'on a confié le développement du bio, avec ses consommateurs en général mal informés, peu engagés, et ses producteurs parfois trop intéressés, n'aboutisse à diluer ou détourner cette autre conception de l'agriculture qu'ils poussent au prix souvent d'une grande abnégation.

Ils se défient des autorités en charge de la régulation des marchés et de leur vision réductrice du bio. Ils citent à cet effet les événements récents et la suppression de la subsidiarité française qui montrent qu'elles pensent le développement des marchés comme une circulation fluide de produits bio homogènes, et pour cela, réduits à un même label, ce qui aboutit invariablement à ramener le bio au plus petit dénominateur commun, «niveler par le bas » et faire fi des exigences plus larges de chacun des cahiers des charges de départ.

Les bio-label renvoient les éco-alternatifs au surcroît d'exigences de leurs chartes privées, aux surcoûts qu'elles impliquent et à leurs circuits spécialisés qui font redouter que le bio ne se transforme en une consommation d'élite. De plus, ce bio cher leur semble ouvrir la voie à une interprétation 
du bio comme un surcroît de qualité des produits justifiant un surprix. Or une telle interprétation ne peut que freiner le développement du bio en exigeant des consommateurs qu'ils consentent à payer plus cher les produits bio. Ils répètent ainsi que, contrairement à un « dogme » répandu, le bio ne coûte pas plus cher que les autres productions :

«... il y a aussi le dogme dans la tête des consommateurs du: de toute façon le bio c'est plus cher, c'est $30 \%$ plus cher, quelqu'un a décidé ça il y a 30 ans, que c'est $30 \%$ plus cher et ça suit quelles que soient les productions, ... Et quand on met 2 bouteilles, une Bio, une pas Bio à côté et qu'on regarde les prix, en général ça ne marche jamais. Ça ne fait pas $30 \%$, mais quelqu'un a décidé que c'était $30 \%$ plus cher et donc c'est un frein »

(Vigneron bio)

Les éco-alternatifs, l'admettent, le bio reste souvent - pas toujours ! - un peu plus cher, mais son achat doit être repensé dans une nouvelle conception de la consommation : fin des gaspillages, plus grande attention portée à ce qui est consommé... une attitude qui compense largement les surcoûts de production et commercialisation. Être bio c'est réfléchir non seulement à la « définition » du bio, mais aussi aux conséquences humaines et économiques de nos actes. C'est cette réflexion généralisée qui doit ramener à une répartition plus juste de la richesse. Les éco-alternatifs se défendent ainsi des accusations d'élitisme en insistant sur le combat politique social qu'ils mènent pour que tout le monde réfléchisse plus loin que «mes goûts », «le prix », et surtout « LE label »... Car cette réduction du bio est une démission du devoir de chacun de réfléchir ; et pour eux, c'est payer bien cher le trafic fluide qui permet au bio de toucher l'ensemble de la planète. Car avec ce label qui « commodifie » le bio ${ }^{10}$ comme disent les économistes, qui en fait une

10. Cet anglicisme désigne la transformation d'un objet en une marchandise, " commodity » en anglais.
« chose » bio d'autant mieux réussie que le label est pensé « crédible » et donc non interrogé, c'est tout le contraire qui se produit. Le label crédible décharge le consommateur de son devoir de penser les conséquences de ses actes et permet à des entreprises d'inventer et mettre en œuvre des solutions bio qui n'en ont pas « l'esprit », comme le bio « hors sol », la bio dite « intensive », ou encore le transport de sols convertis dans d'autres lieux pour l'aménagement de serres bio par exemple.

\section{Bio, qualité globale ou caractéristique objectivée ?}

Est-il possible de réconcilier les frères ennemis ou faut-il les séparer?

«Quand on met une règle on crée les cas limites. C'est un problème! », nous disait un membre de la FNAB. En « objectivant » le bio, le label ouvre la porte à des innovations qui satisfont les critères du label, mais échappent à la procédure collective par laquelle s'élabore et se surveille la qualité globale bio. Leur évaluation est confiée à des clients qui délèguent l'examen du bio à un label qui ne procède qu'à un examen très partiel de la qualité biologique du produit, sourd aux questions de transport, d'eau, d'organisation économique, d'éthique, de justice...

Les bio éco-alternatifs reprochent au label certifié par un tiers de créer la possibilité de son détournement car il permet un usage non « engagé » du bio. Le recours au label peut suspendre l'interrogation critique du bio - ce qu'il est, ses bonnes ou ses mauvaises interprétations, ses ajustements ... pour en faire une qualité déterminée par les critères qui la signalent. Cette objectivation du bio a pour mérite d'en faire une qualité finie, autonome, indépendante du lieu ou de la personne qui l'applique. Si l'on reprend les termes de Latour (1993), la qualité bio est devenue un « mobile immuable », une chose appropriable par chacun et « applicable » à tout objet support 
pour peu que sa fabrication respecte les critères et contraintes du label. Le bio « mis en boîte » par un label acquiert ainsi une existence indépendante des personnes qui l'ont pensé, élaboré. Mais le label autorise aussi une autre transformation. Alors que pour les bio éco-alternatifs, le bio est une qualité globale qui questionne l'ensemble du produit bio, sa fabrication, son transport, sa consommation, son recyclage par exemple, il devient par le fait du label une qualité circonscrite qui peut être intégrée - plus ou moins facilement - aux autres qualités du produit objectivées ou non, attendues ou imposées par le producteur fabricant, les acheteurs ou les régulations marchandes.

Les mises en œuvre du bio qu'ils défendent doivent être selon eux soumises à une évaluation ex post intégrative. Elle doit s'appuyer sur l'ensemble du processus de fabrication, distribution, consommation dès lors qu'il peut avoir une incidence sur la qualité «bio »du produit pensée non plus comme un ensemble de critères, mais l'élaboration toujours en cours d'une alternative à l'impasse de l'agriculture conventionnelle.

La qualité bio qui ressort de cet usage collectif n'est pas « quelque chose » d'autonome ou de prédéfini. Elle est le résultat de multiples mises en œuvre, chacune inséparable de sa situation concrète d'usage et d'une interprétation de ce que le bio peut et doit être, et toutes soumises à une discussion critique animée par d'autres personnes elles aussi engagées dans l'élaboration de l'alternative bio. Que ce soit pour produire, trouver et consommer ces produits, les éco-alternatifs insistent sur la nécessité faite à chacun de s'informer, de recueillir des jugements, de s'enquérir de la validité de ces jugements, d'appendre soi-même à les juger et ainsi de contribuer au travail collectif de critique et d'élaboration de cette qualité globale bio. Le signe ou la certification qui fonde l'acquisition de la qualité bio dans le premier cas, constitue une vision « réductrice », incomplète et parfois même inadaptée dans le second. Il perd sa capacité à signaler et devient objet de discussion et de jugement.

\section{Un bio bifide ?}

Le bio en train de s'élaborer ne peut faire l'objet d'un contrôle de conformité par un tiers. Les associations et notamment Nature \& Progrès qui s'oppose depuis 1995 au label $\mathrm{AB}$ proposent une alternative ; elles ont inventé et mis au point des techniques de garantie participatives, qui ne fonctionnent pas par délégation à des tiers indépendants, mais à l'inverse, par la soumission de tous les projets de production bio de leurs adhérents à la critique collective par les membres engagés et vigilants de l'association (May, 2008 ; Darlong, 200 8; Fonseca et al., 2008 ; IFOAM, 2007).

Dans les entretiens, les associations NP ou Déméter étaient décrites comme des collectifs fermés et sectaires et leurs procédures internes de contrôle, décriées pour leur manque de «transparence », « d'indépendance » et de « garantie objective ». Certes, les procédures de vigilance de l'engagement sont internes et animées par leurs membres, les seuls qu'elles reconnaissent compétents pour cette tâche. L'absence de critères définissant a priori le bio n'est pas un manque de transparence ; c'est la conséquence d'une impossibilité, celle qu'il y a à expliciter définitivement le bio, en tenant compte de tous les possibles, c'est-à-dire quelles que soient la région, la production, l'exploitation et... l'avenir. Et le manque d'indépendance des juges est au contraire une garantie de leur compétence, du partage des idées, de la discussion des projets de production. La compétence des juges du bio vu comme qualité globale en élaboration est indissociable de leur participation à l'élaboration même de cette qualité. La notion d'indépendance n'a pas de sens lorsqu'il s'agit d'évaluer l'interprétation d'une idée ou d'un concept, de même que celle de « garantie objective », car elles supposent 
toutes deux que l'idée ou le concept ait une existence définie et objectivable ${ }^{11}$.

Faut-il alors remplacer le label AB et son contrôle indépendant par une procédure critique participative généralisée ? Cette situation est courante ; les consommateurs, lorsqu'une « qualité » particulière ou un objet les intéresse, interrogent, s'informent, voire se livrent à de véritables « enquêtes » pour faire leurs choix ; ils utilisent et contribuent ainsi à la critique collective qui accompagne la circulation marchande de ces produits. On en connaît aussi les limites. Un investissement fort d'un grand nombre d'acheteurs est nécessaire ; leur acquisition d'informations, la circulation et la confrontation des jugements limitent les possibilités de développement et d'extension de ces marchés. Les certifications proposent au contraire de prendre en charge cette enquête et d'en soulager les consommateurs, afin de faciliter l'intéressement de nouveaux consommateurs et ainsi l'extension et la croissance des marchés. La taille modeste ${ }^{12}$ des associations qui se démarquent du label $\mathrm{AB}$ est en général associée aux exigences de leurs cahiers des charges. Mais ce point ne concerne pas les consommateurs ; le coût faible de l'adhésion aux associations ${ }^{13}$ semble difficilement pouvoir être un facteur limitant important. Il faut vraisemblablement plutôt voir leur petite taille comme une conséquence de la mise en œuvre d'une étroite vigilance qui privilégie les petits projets, à taille humaine, les réseaux courts où l'on peut encore s'informer et où le contrôle participatif peut avoir lieu et rester efficace. Mais lorsque les produits parcourent la planète, lorsqu'une exploitation vend des centaines de produits différents, ou des volumes considérables, les adhérents ne suffisent plus.

11. Pour une discussion complète de l'objectivité d'un contrôle engagé ou dégagé voir (Teil, 2001)

12. En France, Nature \& Progrès compte environ 350 producteurs et 20000 sont certifiés label $\mathrm{AB}$. 13. Pour les consommateurs le coût de l'adhésion est le plus souvent de 10 à 20 euros.
Est-on dès lors condamné à un bio bifide, comme le suggère l'idée de «bifurcation » (Coombes et Campbell, op. cit.) (Campbell et Liepins, op. cit.), avec ses réseaux courts et le contrôle participatif de tous les acteurs, ses marchés locaux et sa taille humaine d'un côté, et de l'autre ses marchés internationaux, des labels internationaux délégués à des organismes contrôleurs indépendants et des produits bio à l'identité floue ?

\section{Le bio durable Une finalité active et cadrée}

L'idée de bifurcation suggère une séparation en deux voies étanches, où le bio " écoalternatif » reste hors de portée et protégé du bio « objectivé » et échappe ainsi à la conventionalisation.

Il ne faut tout d'abord pas se méprendre : ces deux «bio » ne sont pas deux versions différentes et homonymes du bio. Il n'existe pas d'un côté un bio qui serait « éco-alternatif » et de l'autre un autre bio qui serait « objectivé », pas plus que ces deux bio sont le résultat de deux usages de producteurs engagés dans la réflexion bio d'un côté, dans la rentabilité commerciale de l'autre. Ce sont deux régimes d'action qui font émerger des modes de présence différents du bio, l'un sous une forme réduite à des critères, l'autre comme un objet toujours en train de se faire. Chaque régime recourt à des instruments différents de preuve ou d'évaluation de la présence de l'objet, mais qui ne sont jugés incompatibles que dans la mesure où les acteurs estiment que les mises en œuvre de l'un et l'autre sont trop différentes pour continuer à cohabiter sans se nuire sous le même nom. C'est bien cette question que pointe la menace d'usure ou de conventionalisation soulevée par les écoalternatifs. Une séparation entre les deux régimes est-elle opportune ?

Le bio éco-alternatif éprouverait toujours les mêmes peines à développer et allonger ses circuits denses et informés. De son côté le bio objectivé, comme toutes les normes, devrait faire l'objet de constantes révisions, 
additions et ajustements pour s'adapter aux incessants changements et aleas, et assurer sa durabilité.

Chercher à protéger le bio éco-alternatif en le dotant de critères plus nombreux, plus objectifs, comme le conseillent (Conner, 2004), (Guthman, 2004) (Darnhofer et al., op. cit.), comme l'ont fait depuis longtemps Nature \& Progrès ou Déméter permet de recadrer les débordements du mouvement bio en lui donnant de nouvelles limites. Cependant, ce procédé reproduit toujours, comme toute garantie de moyens ou de résultats, la divergence entre le bio vu comme finalité ou comme objet prédéfini. Elle n'est donc pas « La » solution, mais une étape du processus par lequel le bio ne cesse de se penser et de se réviser. Car comme tout ensemble de critères ou contraintes qui ne saisit qu'imparfaitement et provisoirement un objet en devenir tel que le bio, il finit toujours par être débordé par l'arrivée de nouveaux intéressés ; de nouvelles explicitations et ajustements du bio doivent alors à nouveau tenter de le recadrer $^{14}$.

Ce débordement et recadrage n'est pas dû à des approximations des critères et contraintes ; il est constitutif de la pérennisation du bio qui fait tenir deux modalisations différentes de son existence, selon la finalité et l'objectivation en critères, l'une avec l'autre, car elles ne sont rien l'une sans l'autre. Le bio objectivé ne serait rien si le contrôle critique exercé par le bio éco-alternatif ne venait guider et assurer sa crédibilité ; réciproquement, ce dernier décuple ses capacités de développement s'il peut profiter de la capacité à enrôler du bio objectivé. Ils forment tous deux les deux « piliers » qui lui donnent son attractivité et sa résistance.

À mesure de la transformation ou de la

14. Nous reprenons ici l'image très pertinente utilisée par Callon pour décrire la succession des mouvements de pause et de mise en cause qui assurent la durabilité des collectifs (Akrich et al., 2010). diversification de la notion de bio, les signes du bio objectivé doivent être réajustés pour continuer de bénéficier de la crédibilité de la critique. Ils le sont par renforcement des chartes et critères, dans le cas de Nature \& Progrès et Déméter, ou par création de nouveaux cahiers des charges $^{15}$. Leurs divergences signalées par une variété de chartes pluralisent la notion de bio ; elles amènent surtout une prise de distance croissante avec la certification qui borde cette notion. Or c'est bien elle qui doit aussi être ajustée et révisée. C'est le même processus d'échange entre une vision globale et son interprétation réduite qui doit donc être entretenu au sein de l'ensemble des adhérents et non seulement au sein de sous-groupes, comme le préconisent les chercheurs ci-dessus pour protéger des producteurs de dérives amenées par les innovations apportées par le label et jugées inacceptables.

$$
\text { * } *
$$

Comment aider au développement et à la pérennisation de l'agriculture biologique ? Sont-ils aujourd'hui menacés comme le disent de nombreux agrobiologistes et chercheurs ? Faut-il comme eux chercher à assurer la pérennité du bio par une connaissance toujours plus approfondie de ce qu'il est ? Cette idée suppose que le bio est un objet fini, qu'il existe en dehors de ceux qui contribuent chaque jour à le faire exister. Elle est condamnée à se heurter aux évolutions historiques qui en périment l'une après l'autre les définitions. Partant de la conception inverse qui analyse le bio comme le résultat du travail de conception et de mise en œuvre par les acteurs eux-mêmes, nous avons tenté d'esquisser la solution originale qu'ils apportent aux problèmes que peut poser la diversification d'un mouvement en croissance.

15. C'est le cas en France de la nouvelle marque Bio-Cohérence. 
Comme le souligne la sociologie de la traduction ${ }^{16}$, le slogan «Traduttore, traditore » reprend à merveille les difficultés que doivent affronter nombre de mouvements qui veulent se développer. La traduction, c'est-à-dire l'enrôlement de nouveaux adeptes qui accompagne le grossissement d'un mouvement ou la diffusion de connaissances ou de techniques, amène toujours de nouvelles interprétations, de nouvelles mises en œuvre de pratiques et une diversification des objectifs ou du message. Vues du cadre étroit des interprétations individuelles ou stabilisées, ce sont autant de dérives et de coups portés à leur "véritable » signification, mais aussi d'ajustements qui leur permettent de convaincre de plus larges publics, de se développer et de durer. Ainsi le développement d'un mouvement semble souvent devoir se payer d'une «pluralisation » de son message, au travers des nouvelles interprétations apportées par les nouveaux adeptes, qui en fait la force mais aussi la faiblesse.

La solution qui semble émerger consiste à faire tenir ensemble, et c'est là toute la difficulté, deux régimes de présence du bio.

Le label forme d'un côté une certification qui introduit une objectivation de la qualité bio. Mise en place pour assurer le développement du bio elle apporte des interprétations parfois innovantes dont l'évaluation échappe aux intéressés les plus investis, pour reposer sur les producteurs ou les consommateurs dont l'engagement dans le développement d'une agriculture bio pérenne n'est pas assuré. Des associations réagissent alors en mettant en place des marques privées et un durcissement du cahier des charges assorti d'une autre conception du bio non pas comme des critères délimitant une qualité, mais comme un cadre minimal bordant l'élaboration du bio saisi comme un but ou une qualité

16. Cf. Law et Williams, (1982); Callon et al. (1983) ; Latour (1984) ou le recueil Akrich et al. (2006) pour une réédition des textes fondateurs. globale non prédéfinie et s'étendant sans limite a priori à tous les compartiments de la production, de la distribution et de la consommation. Et c'est grâce à une vigilance critique interne qu'elles produisent l'ajustement et la révision permanente du bio pour le rendre pérenne ou durable. Ces associations ajoutent toutefois une contrainte forte de participation active à la discussion du bio.

Le bio apparaît dès lors comme un objet mêlant des conceptions très opposées et concurrentes. Plutôt que de les distinguer ou de délaisser l'une ou l'autre de ces conceptions, nous avons défendu l'idée du nécessaire maintien de leurs interactions de sorte qu'il puisse profiter des capacités de développement apportée par la qualité bio objectivée dans une certification et que les innovations qu'elle apporte soient validées par la discussion critique du bio comme qualité globale et non par le simple respect de critères a priori.

Finalement, la crainte de la conventionalisation apparaît comme l'un des questionnements ${ }^{17}$, celui des bio éco-alternatifs, qui interroge la bonne coexistence des deux régimes. Tant qu'elle ne conclue pas à la nécessité de rompre entre deux « visions » incompatibles et de leur attribuer deux noms différents - ce qui arrive dans d'autres cas de certifications (Teil, 2011), elle contribue à leurs interactions mutuelles.

17. Les bio « label » en soulèvent une autre symétrique, qui pointe le confinement du bio au collectif critique interne des associations. 


\section{RÉFÉRENCES BIBLIOGRAPHIQUES}

Akrich M., Barthe Y., Muniesa F., Mustar P. (eds.) (2010). Débordements. Mélanges offerts à Michel Callon. Paris, Presses de l'École des Mines.

Akrich M., Callon M., Latour B. (2006). Sociologie de la traduction : textes fondateurs. Paris, Presses de l'École des Mines.

Best H. (2008). Organic agriculture and the conventionalization hypothesis: a case study from West Germany. Agriculture and Human Values, vol. 25, $\mathrm{n}^{\circ} 1$, p. 95 106.

Buck D., Getz C., Guthman J. (1997). From Farm to Table: the Organic Vegetable Commodity Chain of Northern California. Sociologia Ruralis, vol. 37, n 1, p. 3-20.

Callon M., Courtial J.-P., Turner W.-A., Bauin S. (1983). From Translation to Problematic Networks : an Introduction to Co-word Analysis. Social Science Information, $n^{\circ} 22$, p. 191-235.

Campbell H., Liepins R. (2001). Naming Organics: Understanding Organic Standards in New Zealand as a Discursive Field. Sociologia Ruralis, vol. 41, $\mathrm{n}^{\circ} 1$, p. 22-39.

Conner D. (2004). Beyond organic: information provision for sustainable agriculture in a changing market. Journal of Food Distribution Research, vol. 35, $\mathrm{n}^{\circ} 1$, p. 34-39.

Coombes B., Campbell H. (1998). Dependent Reproduction of Alternative Modes of Agriculture: organic Farming in New Zealand. Sociologia Ruralis, vol. 38, $\mathrm{n}^{\circ} 2$, p. 127-45.

Dantsis T., Loumou A., Giourga C. (2009). Organic Agriculture's Approach towards Sustainability; Its Relationship with the Agro-Industrial Complex, A Case Study in Central Macedonia, Greece. Journal of Agricultural and Environmental Ethics, vol. 22, n 3, p. 197-216.

Darlong V. (2008). Harmonizing jhum (shifting cultivation) with PGS organic stan- dards in Northeast India: key features and characteristics of jhum for process harmonization. Cultivating the future based on science, vol. 1, "Organic Crop Production”. Proceedings of the Second Scientific Conference of the International Society of Organic Agriculture Research (ISOFAR), held at the 16th IFOAM Organic World Conference in Cooperation with the International Federation of Organic Agriculture Movements (IFOAM) and the Consorzio ModenaBio in Modena, Italy, 18-20 June, p. 718-21.

Darnhofer I. (2006). Organic farming between professionalisation and conventionalisation- The need for a more discerning view of farmer practices. In «The Organic Congress», 2. Odense DK.

Darnhofer I., Lindenthal T., Bartel-Kratochvil R. Zollitsch W. (2010). Conventionalisation of organic farming practices: from structural criteria towards an assessment based on organic principles. A review. Agron. Sustain. Dev., vol. 30, $\mathrm{n}^{\circ} 1$, p. 67-81.

Déméter, 2004, Cahier des Charges. Identification des produits.

Fonseca M.-F.-d.-A.-C., Wilkinson J., Egelyng H., Mascarenhas G.-C.-C. (2008). The institutionalization of Participatory Guarantee Systems (PGS) in Brazil: organic and fair trade initiatives. Cultivating the future based on science, vol. 2, "Livestock, socio-economy and cross disciplinary research in organic agriculture". Proceedings of the Second Scientific Conference of the International Society of Organic Agriculture Research (ISOFAR), held at the 16th IFOAM Organic World Conference in Cooperation with the International Federation of Organic Agriculture Movements (IFOAM) and the Consorzio ModenaBio in Modena, Italy, 18-20 June, p. 368-71. 
Guthman J. (2004). The Trouble with "Organic Lite" in California: a Rejoinder to the "Conventionalisation" Debate. Sociologia Ruralis, vol. 44, n 3, p. 30116.

Hall A., Mogyorody V. (2001). Organic Farmers in Ontario: an Examination of the Conventionalization Argument. Sociologia Ruralis, vol. 41, n ${ }^{\circ}$, p. 399-22.

Harrouch A. (2003). Le rôle de la Nature et Progrès dans l'histoire de la bio en France: témoignage d'une actrice engagée. Nature \& Progrès, $\mathrm{n}^{\circ} 44$, novembre-décembre.

IFOAM. (2007). Systèmes de garantie participatifs : vision idéale. $\mathrm{n}^{\circ} 3$.

IFOAM. (non daté). Principles of organic agriculture-Préambule.

James W. (1996). Essays in radical empiricism. 1ère édition (1912), New York, ed. University of Nebraska Press \& Longmans Greens and Co., 283 p.

Latour B. (1984). Les microbes : guerre et paix, suivi de Irréductions. Paris, A.-M. Métailié, Latour, B. (1993). « Le topofil de Boa-Vista ». Raisons Pratiques, $\mathrm{n}^{\circ} 4$ : 187-216.

Latour B. (2007). Changer de société, refaire de la sociologie. Paris, La Découverte, Poche.

Law J., Williams R. J. (1982). Putting Facts Together. Social Studies of Science, $\mathrm{n}^{\circ} 12$, p. 535-58.

Lockie, S., Halpin, D., 2005. The "Conventionalisation" Thesis Reconsidered: Structural and Ideological Transformation of
Australian Organic Agriculture. Sociologia Ruralis, $\mathrm{n}^{\circ}$ 45, 284-307.

May C. (2008). Petit guide des SPG ou comment développer et faire fonctionner les systèmes participatifs de garantie. Allemagne, IFOAM, $36 \mathrm{p}$.

Michelsen J. (2001). Recent Development and Political Acceptance of Organic Farming in Europe-Introduction au numéro. Sociologia Ruralis, vol. $41, \mathrm{n}^{\circ} 1$, p. 3-20.

Murdoch J., Miele M. (1999). "Back to Nature": changing "Worlds of Production" in the Food Sector. Sociologia Ruralis, vol. 39, $\mathrm{n}^{\circ}$ 4, p. 465-83.

Rosin C., Campbell H. (2009). Beyond bifurcation: examining the Conventions of Organic Agriculture in New Zealand. Journal of Rural Studies, vol. 25, $\mathrm{n}^{\circ} 1$, p. 35-47.

Teil G. (2001). La production du jugement esthétique sur les vins par la critique vinicole. Revue de Sociologie du Travail, vol. 43, $\mathrm{n}^{\circ} 1$, p. 67-89.

Teil G. (2011) No such thing as terroir? Objectivities and the Regimes of Existence of Objects. Science technology and Human Values, p. 21.

Teil G., Barrey S., Floux P., Hennion A. (2011). Le vin et l'environnement : faire compter la différence. Paris, Presses de l'Ecole des Mines, 327 p.

Tovey H. (1997). Food, Environmentalism and Rural Sociology: on the Organic Farming Movement in Ireland. Sociologia Ruralis, vol. 37, ${ }^{\circ} 1$, p. 21-37. 
ANNEXES

\section{Détails de la répartition des entretiens de l'enquête}

Annexe 1. Les vignerons

\begin{tabular}{c|l|r|r|c}
\hline \multirow{2}{*}{ Type d'exploitation } & \multicolumn{2}{|c|}{ Type de qualité environnementale } & \multicolumn{3}{|c}{ Nombre } \\
\cline { 3 - 5 } & & V. de L. & LR & Jura \\
\hline Coopérative & Sans & 1 & & \\
\hline Coopérative & Certification de qualité industrielle & 1 & 2 & \\
\hline Coopérative & Viticulture raisonnée & 1 & 3 & \\
\hline Coopérative & Pourcentage de la production en bio & 2 & & \\
\hline Exploitant viticole & Sans & 8 & 1 & \\
\hline Exploitant viticole & Certification de qualité industrielle & 4 & & \\
\hline Exploitant viticole & Viticulture raisonnée & 7 & 5 & \\
\hline Exploitant viticole & Viticulture raisonnée non certifiée & 5 & 5 & \\
\hline Exploitant viticole & Label AB & 26 & 14 & \\
\hline Exploitant viticole & Label de biodynamie & 13 & 5 & \\
\hline Exploitant viticole & Biodynamie non certifiée & 4 & & \\
\hline Exploitant viticole & Vin naturel ou de terroir & 2 & 4 & 2 \\
\hline & Total & 68 & 45 & 2 \\
\cline { 2 - 5 } & Total & & 115 & \\
\hline
\end{tabular}

Les producteurs en biodynamie sont aussi bio. Nous n'avons compté que la qualification biodynamie. Expl. Viticole : Exploitant viticole

VdL. : Val de Loire

LR : Languedoc-Roussillon

VR : Viticulture raisonnée

bio : Agriculture biologique

bd : Agriculture biodynamique

ssl : sans certification

CQ : Certification de Qualité Industrielle

$\%$ bio : Coopérative commercialisant une partie de sa production en bio

VNT : vin naturel ou de terroir, revendiquant un nécessaire retour à une qualité plus authentique, mais n'ayant pas de certification environnementale 
Analyse du débat autour du label bio

Annexe 2. L'ensemble de l'échantillon

\begin{tabular}{|c|c|c|}
\hline Domaine d'activité & & Nombre \\
\hline \multirow[t]{3}{*}{ Producteurs } & & 115 \\
\hline & dont certifiés bio & 62 \\
\hline & dont engagés bio & 10 \\
\hline \multirow[t]{7}{*}{ Distribution Vente } & Grande distribution & 9 \\
\hline & Négoce & 1 \\
\hline & Caviste & 8 \\
\hline & Boutique coopérative bio & 5 \\
\hline & Boutique associative bio & 1 \\
\hline & Boutique franchisée bio & 1 \\
\hline & Caviste \% QE & 11 \\
\hline \multirow[t]{3}{*}{ Administration centrale } & Ministère de l'agriculture & 6 \\
\hline & Agence bio & 2 \\
\hline & Administration $\mathrm{AOC}$ & 4 \\
\hline \multirow[t]{2}{*}{ Industrie phytosanitaire } & Firme phytosanitaire & 5 \\
\hline & Distributeur produits phytosanitaires & 1 \\
\hline \multirow[t]{4}{*}{ Technique \& Recherche } & Encadrement technique & 6 \\
\hline & Enc. Tech. bio & 3 \\
\hline & Recherche & 4 \\
\hline & Enseignement agricole & 1 \\
\hline Syndicat agricole & Syndicat agricole & 7 \\
\hline \multirow[t]{4}{*}{ Certification de qualité } & Certification VR & 4 \\
\hline & Certification CQ & 1 \\
\hline & Certification bio & 1 \\
\hline & Syndicat $A O C$ vin & 9 \\
\hline Restauration & Restaurateur bio & 3 \\
\hline \multirow[t]{5}{*}{ Média } & Critique vinicole & 4 \\
\hline & Presse régionale & 2 \\
\hline & Journaliste bio & 1 \\
\hline & Critique vin naturel & 2 \\
\hline & Presse économique & 1 \\
\hline \multirow[t]{2}{*}{ Consommateur } & Consommateurs 13 & \\
\hline & Total & 231 \\
\hline
\end{tabular}

